\title{
Determinação da demanda hídrica do quiabeiro em Campos dos Goytacazes, $\mathbf{R J}^{1}$
}

\author{
Determination of water requirement of okra in Campos dos Goytacazes, RJ
}

\author{
Herval Martinho Ferreira Paes ${ }^{2}$, Barbara dos Santos Esteves ${ }^{3 *}$ e Elias Fernandes de Sousa ${ }^{2}$
}

\begin{abstract}
Resumo - O objetivo deste estudo foi descrever as necessidades hídricas do quiabeiro em cada estádio do seu desenvolvimento, por meio da determinação da evapotranspiração da cultura e dos coeficientes de cultivo (Kc). $\mathrm{O}$ experimento foi conduzido na Estação Evapotranspirométrica da Universidade Estadual do Norte Fluminense Darcy Ribeiro, no município de Campos dos Goytacazes, RJ. A cultivar utilizada foi a Santa Cruz 47, plantada no espaçamento $0,25 \mathrm{~m}$ x 1,00 m, em uma área de $1024 \mathrm{~m}^{2}$, irrigada por aspersão convencional. Na área foram feitas as determinações da evapotranspiração da cultura e do coeficiente cultural, utilizando-se um lisímetro de $6 \mathrm{~m}^{2}$ com balança eletrônica com variação de peso de $0,58 \mathrm{~kg}$. Os resultados obtidos permitiram quantificar a evapotranspiração da cultura no período em $314 \mathrm{~mm}$. O Kc do quiabeiro no primeiro estádio de desenvolvimento variou entre os valores de 0,8 e 0,5 , obtendo o valor médio de 0,68 ; no segundo estádio variou de 0,5 a 1,0 , sendo a média de 0,79 ; e no terceiro estádio o Kc variou de 1,0 a 0,3 , sendo a média de 0,54 .
\end{abstract}

Palavras-chave - Manejo de irrigação. Evapotranspiração real. Kc. Tensão de água no solo.

\begin{abstract}
This study was carried out to describe the water needs of okra at each stage of its development, through the determination of crop evapotranspiration and crop coefficient $(\mathrm{kc})$ providing subsidies to irrigated agriculture. The experiment was conducted at Station evapotranspirometers Universidade Estadual do Norte Fluminense Darcy Ribeiro in Campos State, Brazil. The cultivar used was the Santa Cruz 47, planted in a spacing of $0.25 \mathrm{mx} 1.00 \mathrm{~m}$ in an area of $1024 \mathrm{~m}^{2}$, irrigated by sprinkler. Area was done to determine the evapotranspiration and Kc, using a lysimeter $6 \mathrm{~m}^{2}$ with an electronic scale with weight change of $0,58 \mathrm{~kg}$. The results allowed to quantify the crop evapotranspiration during the period under $314 \mathrm{~mm}$. The Kc of okra in the first stage of development varied between values of 0.8 and 0.5 , obtaining the average value of 0.68 , the second stage ranged from 0.5 to 1.0 , with a mean of 0,79 , and the third stage Kc ranged from 1.0 to 0.3 , the average being 0.54 .
\end{abstract}

Key words - Irrigation management. Evapotranspiration. Kc. Soil water tension.

\footnotetext{
*Autor para correspondência

'Recebido para publicação em 14/10/2010; aprovado em 06/09/2011

Parte da Dissertação do primeiro autor

2Produção Vegetal, UENF/CCTA/LFIT, Campos dos Goytacazes-RJ, Brasil, E hpaes@uenf.br, efs@uenf.br

'Programa de Pós-Graduação em Produção Vegetal, UENF/CCTA/LEAG, Av. Alberto Lamego 2000,Parque Califórnia, Campos dos Goytacazes-RJ, Brasil, 28.015-620, barbbarase@ yahoo.com.br
} 


\section{Introdução}

A cultura do quiabeiro (Abelmoschus esculentus (L.) Moench) é uma das mais importantes para oEstado do Rio de Janeiro. O quiabo é uma hortaliça da família das Malváceas, um alimento popular de alto valor nutricional, com grande aceitação no mercado, sendo os pequenos produtores os maiores responsáveis por toda a sua produção.

Em função da crescente preferência pelo consumidor, tem-se registrado expressiva expansão da cultura do quiabeiro em todo o Brasil, principalmente nos Estados do Rio de Janeiro, São Paulo e Sergipe (CAVALCANTE et al., 2010). As hortaliças em geral têm o seu desenvolvimento intensamente influenciado pelas condições de umidade do solo. A deficiência de água é, normalmente, o fator mais limitante à obtenção de produtividades elevadas e produtos de boa qualidade.

No que se refere ao manejo de irrigação, a base para a quantificação da água a ser aplicada a uma determinada cultura está comumente associada à capacidade da superfície do solo e da vegetação de perder água para a atmosfera. A forma usual de se quantificar a água a ser aplicada ao longo do ciclo da cultura, é considerar os processos de evaporação do solo e de transpiração da planta conjuntamente, no que se denomina evapotranspiração (SILVA; RAO, 2006). Na agricultura, informações quantitativas da evapotranspiração são de grande importância na avaliação da severidade, distribuição e frequência dos déficits hídricos, elaboração de projetos e manejo de sistemas de irrigação e drenagem (HENRIQUE; DANTAS, 2007).

Osucessona agriculturairrigadadependede inúmeras variáveis que devem ser estudadas antes da implantação de um projeto de irrigação. Para isso, é necessário que se tenham informações precisas sobre vários parâmetros básicos, dentre os quais: a evapotranspiração de referência (ETo), a evapotranspiração da cultura (ETc) e o coeficiente da cultura (Kc) (CHAVES et al., 2005). A ETo pode ser obtida por medidas diretas ou por estimativas (CARDOSO et al., 2005). A determinação direta da evapotranspiração pode ser feita por lisímetros, balanço hídrico e controle de umidade no solo (ACOSTA, 2009). A ETo também pode ser determinada indiretamente por fórmulas empíricas, baseadas em dados meteorológicos. A ETc é obtida através do valor da ETo corrigida pelo Kc, sendo este dependente do tipo de cultura e do estágio de desenvolvimento. $\mathrm{O} \mathrm{Kc}$ é a expressão da demanda hídrica da planta, sendo que este não é valor fixo para todas as situações climáticas. A distribuição temporal dos valores do Kc, durante o ciclo de crescimento da cultura, constitui a curva de cultura (ALLEN et al., 1998). Segundo Medeiros et al. (2004) o Kc é um parâmetro relacionado aos fatores ambientais e fisiológicos das plantas, devendo, preferencialmente, ser determinado para as condições locais nas quais será utilizado.
Váriosestudos sobre consumohídricotais comoPosse et al. (2008) com o mamoeiro, Mendonça et al. (2007) com o feijoeiro, Silva e Rao (2006) com o amendoim e Chaves et al. (2005) com a pimenteira, vem sendo conduzidos em diversos locais do Brasil, em várias culturas. As informações acerca das necessidades hídricas do quiabeiro são escassas na literatura. Dessa forma, o objetivo deste estudo foi descrever as necessidades hídricas do quiabeiro em cada estádio fenológico de seu desenvolvimento, por meio da determinação da evapotranspiração da cultura e dos coeficientes de cultivo em Campos dos Goytacazes, RJ.

\section{Material e métodos}

O experimento foi conduzido na Estação Experimental de Campos dos Goytacazes, PESAGRORIO (coordenadas geográficas de $21^{\circ} 18^{\prime} 47^{\prime \prime}$ de latitude Sul e $41^{\circ} 18$ '24" de longitude Oeste e altitude de $11 \mathrm{~m}$ ), pertencente à Universidade Estadual do Norte Fluminense Darcy Ribeiro (UENF), sendo o plantio realizado no dia 30/04/2002, numa área de $1024 \mathrm{~m}^{2}$, tendo a duração total de 180 dias. Segundo a classificação climática de Köppem, o clima da região é classificado como Aw, isto é, clima tropical úmido, com verão chuvoso, inverno seco e temperatura do mês mais frio superior a $18^{\circ} \mathrm{C}$. O solo da área experimental é um Cambissolo de origem fluvial, pouco profundo, com drenagem moderada a imperfeita. Na Tabelas 1 e 2 estão apresentas as características físicohídricas e a análise química do solo, respectivamente.

As correções no solo foram feitas de acordo com a análise química. A cultivar utilizada foi a Santa Cruz 47, plantada no espaçamento $0,25 \mathrm{~m}$ x 1,00 m, sendo mantida livre das plantas daninhas. A ocorrência de oídio (Erysiphe cichoracearum De Candolle) foi controlada com pulverizações de fungicidas a base de enxofre $80 \%$ (400 g $\left.100 \mathrm{~L}^{-1}\right)$. Outros problemas fitossanitários que atingiram a cultura, como a incidência de pulgão (Aphis gossypii Glov.) e da mosca branca (Bemísia tabaci), foram controladas com revezamento de deltamethrine (30 ml $\left.100 \mathrm{~L}^{-1}\right)$ e imidacloprid $\left(30 \mathrm{~g} 100 \mathrm{~L}^{-1}\right)$, em aplicações semanais no período de maior incidência, e quinzenais

Tabela 1 - Características físico-hídricas do solo da área experimental: Capacidade de campo (CC\%, em peso); Ponto de murcha (PM\%, em peso) e Densidade aparente (DA em $\mathrm{g} \mathrm{cm}^{-3}$ )

\begin{tabular}{cccc}
\hline Profundidade & CC & PM & DA \\
\hline $0-20$ & 12,3 & 6,4 & 1,81 \\
$20-40$ & 15,2 & 10,9 & 1,77 \\
$40-60$ & 17,1 & 11,8 & 1,76 \\
\hline
\end{tabular}


Tabela 2 - Resultados da análise química do solo

\begin{tabular}{ccccccccccccccc}
\hline $\mathrm{pH}$ & $\mathrm{P}$ & $\mathrm{K}$ & $\mathrm{Ca}$ & $\mathrm{Mg}$ & $\mathrm{Al}$ & $\mathrm{H}+\mathrm{Al}$ & $\mathrm{Na}$ & $\mathrm{C}$ & $\mathrm{MO}$ & $\mathrm{SB}$ & $\mathrm{T}$ & $\mathrm{T}$ & $\mathrm{m}$ & $\mathrm{V}$ \\
\hline & $\mathrm{MG}$ & $\mathrm{dm}^{-3}$ & & & $\mathrm{cmol}_{\mathrm{c}}$ & $\mathrm{dm}^{-3}$ & & $\%$ & $\mathrm{gm}^{-3}$ & $\mathrm{cmol}_{\mathrm{c}}$ & $\mathrm{dm}^{-3}$ & & & $\%$ \\
5,3 & 15 & 29 & 1,2 & 0,5 & 0,0 & 3,2 & 0,10 & 0,59 & 10,2 & 1,9 & 5,1 & 1,9 & 0 & 37 \\
\hline
\end{tabular}

Sendo $\mathrm{SB}=$ soma de bases; $\mathrm{T}=\mathrm{CTC}$ a pH 7,0; $\mathrm{t}=$ a CTC efetiva; $\mathrm{m}=$ saturação de alumínio e $\mathrm{V}=$ saturação por bases

fora deste período. $\mathrm{Na}$ área do lisímetro, ocorreu grande incidência de nematóides (Meloidogyne sp), provenientes de um plantio anterior ao trabalho de pesquisa, o que ocasionou a morte de algumas plantas.

Para determinação da evapotranspiração de cultura (ETc) utilizou-se um lisímetro de balança com $6 \mathrm{~m}^{2}$, em chapa de aço, com túnel de acesso a base da balança. O lisímetro possui balança eletrônica marca J. Star, modelo 210, que permite a leitura de variação de peso de $0,58 \mathrm{~kg}(0,09 \mathrm{~mm})$, onde foi instalado um datalogger XPTO, modelo CD 10 Mega, que armazenou dados de leitura do lisímetro a cada vinte minutos, os quais foram transferidos para um computador com programação específica. $\mathrm{O}$ visor da balança e o datalogger foram instalados em um abrigo para a proteção contra a umidade, sendo interligados a uma bateria de $12 \mathrm{~V}$, de maneira que, mesmo na ausência de energia elétrica, a coleta de dados fosse mantida. O coeficiente cultural $(\mathrm{Kc})$ foi obtido diariamente, sendo efetuadas médias semanais para um melhor entendimento da demanda hídrica da cultura do quiabeiro, sendo utilizada a Equação 1.

$$
\mathrm{Kc}=\frac{\mathrm{ETc}}{\mathrm{ETo}}
$$

De um modo geral, os valores de Kc para as hortaliças podem ser divididos em quatro estádios de desenvolvimento, sendo relativamente simples este procedimento. Porém, neste estudo estão apresentados apenas três estádios, com seus respectivos coeficientes culturais, pois o terceiro estádio inicia-se no inicio do período da floração e prolonga-se até o final da produção de frutos verdes e comerciais.

A evapotranspiração de referência (ETo) foi estimada pelo método de Penman-Monteith parametrizado, proposto por Allen et al. (1998), de acordo com a Equação 2:

$$
\mathrm{EToPM}=\frac{0,408 \Delta(\mathrm{Rn}-\mathrm{G})+\gamma \frac{900}{\mathrm{~T}+273} \mathrm{U}_{2}(\text { es- ea })}{\Delta+\gamma\left(1+0,34 \mathrm{U}_{2}\right)}
$$

em que: EToPM é a evapotranspiração potencial, em $\mathrm{mm} \mathrm{d}^{-1}$; Rn é o saldo de radiação, em $\mathrm{MJ} \mathrm{m}^{-2} \mathrm{~d}^{-1}$; G é o fluxo de calor no solo, em $\mathrm{MJ} \mathrm{m}^{-2} \mathrm{~d}^{-1}$; $\mathrm{T}$ é a temperatura média diária do ar, em ${ }^{\circ} \mathrm{C} ; \gamma$ é a constante psicrométrica, em $\mathrm{kPa}^{\circ} \mathrm{C}^{-1} ; \Delta$ é a tangente da curva de pressão de saturação de vapor em função da temperatura do ar em $\mathrm{kPa}^{\circ} \mathrm{C}^{-1} ; \mathrm{U}_{2}$ é a velocidade média diária do vento a $2 \mathrm{~m}$ de altura, em $\mathrm{m} \mathrm{s}^{-1} ;\left(\mathrm{e}_{\mathrm{s}}-\mathrm{e}_{\mathrm{a}}\right)$ é o déficit de pressão de vapor, em $\mathrm{kPa}$.

A área foliar média da cultura foi verificada semanalmente a partir da coleta de cinco plantas da área experimental. Os dados climáticos utilizados foram obtidos a partir da estação automática instalada a poucos metros do local, da marca Thies Clima, modelo DL-15. O manejo da irrigação foi efetuado a partir de sensores de resistência elétrica (sensores Watermark 645WD, da empresa Spectrum Technologies Inc., com medida de potencial hídrico de 0 a $200 \mathrm{Kpa}$, conectados a um datalogger Watch Dog modelo 200); os mesmos foram instalados a $15 \mathrm{~cm}$ e a $45 \mathrm{~cm}$ de profundidade, sendo o de $15 \mathrm{~cm}$ o de decisão do momento de irrigar, enquanto o de $45 \mathrm{~cm}$ o de controle da profundidade da lâmina aplicada (SAAD; LIBARDI, 1992). Sugere-se que os watermarkers sejam instalados em duas profundidades: $1 / 3$ e $2 / 3$ da profundidade efetiva do sistema radicular das plantas, com o controle das irrigações baseado na média das duas leituras (MAROUELLI et al., 1996). A irrigação era realizada quando o sensor de $15 \mathrm{~cm}$ atingia as proximidades de $30 \mathrm{Kpa}$. O sistema de irrigação utilizado foi o de aspersão convencional, sendo programado para ocorrer no período das 0:00 às 3:00 horas, por um sistema automatizado marca Galcon, modelo Yarden Super 8. A drenagem do lisímetro, também automatizada, foi programada para ocorrer no mesmo período da irrigação, por intermédio de solenóides e válvulas de controle hidráulico.

A profundidade efetiva das raízes, fator importante para o manejo da irrigação, foi acompanhada nas diversas fases de desenvolvimento da cultura. Para tal foi aberta uma trincheira em uma das linhas de plantio, onde se instalou um vidro ligeiramente inclinado em uma de suas paredes, devidamente protegido com plástico preto (para evitar a luminosidade), e com folhas de coqueiro (para evitar aquecimento do plástico), de modo a se poder observar a profundidade efetiva das raízes regularmente, sendo os dados apresentados na Tabela 3. 
Tabela 3 - Profundidade das raízes do quiabeiro durante o experimento

\begin{tabular}{ccc}
\hline \multirow{2}{*}{ Dias após plantio } & Profundidade & Estádio da cultura \\
\cline { 2 - 3 } & efetiva $(\mathrm{cm})$ & 1 \\
\hline 6 & 5 & 1 \\
20 & 8 & 1 \\
28 & 10 & 1 \\
39 & 15 & 1 \\
45 & 20 & 1 \\
50 & 24 & $1-2$ \\
60 & 40 & $2-3$ \\
70 & 50 & 3 \\
78 & 60 & 3 \\
94 & 70 & \\
\hline
\end{tabular}

\section{Resultados e discussão}

Os dados climatológicos obtidos junto à Estação meteorológica onde foi realizado o experimento estão apresentados na Tabela 4.

A temperatura média, a umidade relativa e a velocidade do vento, para os meses em que ocorreu o experimento, ficaram próximas às médias mensais obtidas por Esteves et al. (2010), para um período de 10 anos, na mesma região. $\mathrm{O}$ período no qual o experimento foi conduzido é caracterizado como período de inverno. $\mathrm{Na}$ Figura 1 estão apresentadas as precipitações ocorridas e as irrigações realizadas durante o período experimental.

Aprecipitação total durante operíodo do experimento foi de $299,7 \mathrm{~mm}$ e a lâmina irrigada foi de $221 \mathrm{~mm}$, totalizando uma lâmina total de $520,7 \mathrm{~mm}$. A tensão média para a profundidade de $15 \mathrm{~cm}$ foi de $20 \mathrm{kPa}$. Na camada
Figura 1 - Precipitação + irrigação, durante o período experimental

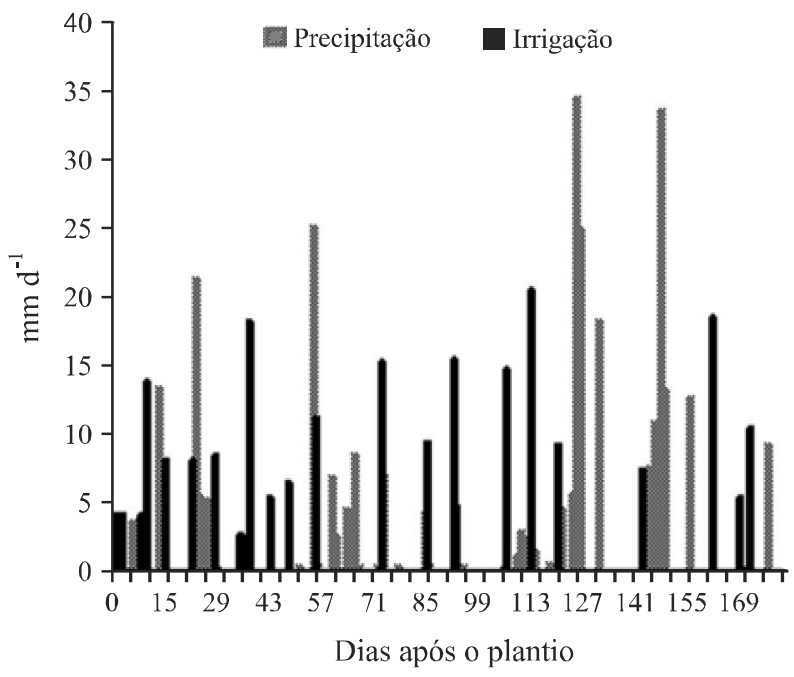

de $45 \mathrm{~cm}$, a tensão média foi de $27 \mathrm{kPa}$, com exceção dos períodos de inicio e do final do experimento. Paes (2003) afirma que o quiabeiro é uma cultura altamente sensível ao déficit hídrico, sendo de $30 \mathrm{kPa}$ o valor máximo de tensão de água no solo para se promover a irrigação. Portanto, de acordo com as tensões médias das profundidades de $15 \mathrm{e}$ $45 \mathrm{~cm}$, a umidade do solo foi sempre mantida em níveis elevados. Em culturas como berinjela, estudada por Bilibio et al. (2010) e tomate, estudado por Marouelli e Silva (2006), as tensões criticas para a maior produtividade foram $15 \mathrm{kPa}$ e $10 \mathrm{kPa}$, respectivamente, sendo estas culturas mais sensíveis ao déficit hídrico que o quiabeiro.

Valores médios semanais (média de 7 dias) foram utilizados para estimar a ETc e a ETo, tal qual em Posse et al. (2008) para o mamoeiro, durante todo o período de desenvolvimento da cultura do quiabeiro, sendo apresentadas na Figura 2.

Tabela 4 - Valores totais e médios mensais da ETo (mm d-1), precipitação (mm mês-1), temperatura média do ar T $\left({ }^{\circ} \mathrm{C}\right)$, umidade relativa média do ar (UR\%) e velocidade do vento a $2 \mathrm{~m}(\mathrm{VV})$, para o período de estudo

\begin{tabular}{lccccc}
\hline \multicolumn{1}{c}{ Meses } & ETo & Precipitação & T $\left({ }^{\circ} \mathrm{C}\right)$ & UR\% & VV \\
\hline Maio & 3,37 & 52,6 & 23,3 & 76,5 & 1,7 \\
Junho & 2,95 & 35,1 & 22,9 & 80,5 & 1,5 \\
Julho & 2,64 & 24,1 & 20,3 & 77,7 & 1,5 \\
Agosto & 3,92 & 19,8 & 22,3 & 76,0 & 2,2 \\
Setembro & 3,41 & 146,5 & 20,6 & 77,7 & 2,4 \\
Outubro & 5,50 & 21,6 & 24,0 & 74,3 & 2,8 \\
Totais/médias & 3,62 & 299,7 & 22,8 & 77,1 & 2,0 \\
\hline
\end{tabular}


Figura 2 - Valores médios semanais da evapotranspiração da cultura (ETc) e da evapotranspiração de referência (ETo) em função dos dias após o plantio do quiabeiro

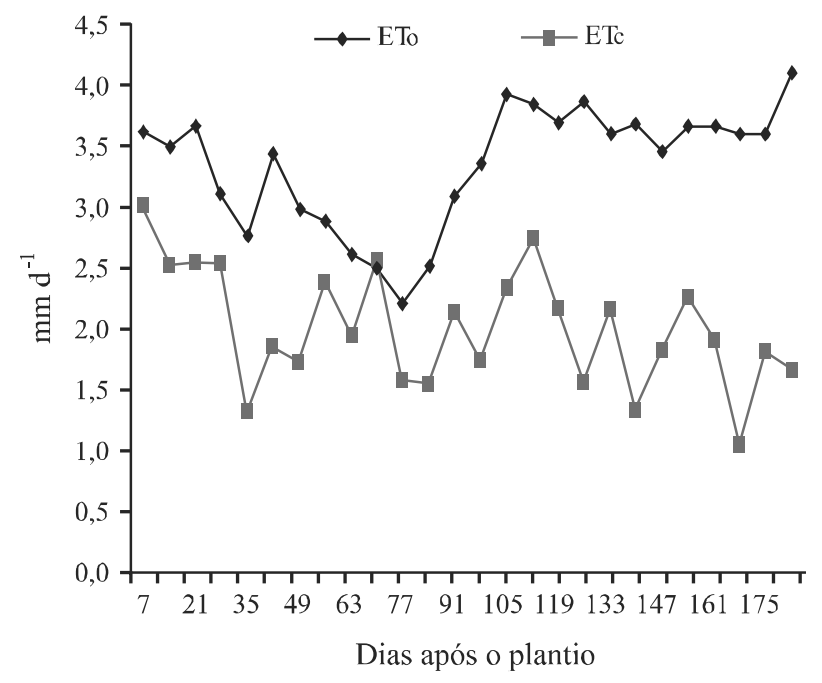

A taxa média da ETo foi de $3,62 \mathrm{~mm} \mathrm{~d}^{-1}$, totalizando $658,6 \mathrm{~mm}$ durante o ciclo. A ETc total obtida durante o ciclo do quiabeiro foi de $314,0 \mathrm{~mm}$, sendo a taxa média da ETc de $1,73 \mathrm{~mm} \mathrm{~d}^{-1}$.

Observou-se que o $\mathrm{Kc}$ possui valores iniciais próximos de 0,8 e decai a valores próximos de 0,5 nos primeiros 40 dias após o plantio (DAP). Verifica-se que este fato ocorre no período do Estádio 1, período de estabelecimento da cultura, o qual termina quando a cobertura foliar das plantas atinge $10 \%$ da área a ser coberta. $\mathrm{O}$ valor de 0,8 pode ser explicado pela ausência de cobertura vegetal, o que proporcionou altas taxas de evaporação do solo devido à alta frequência de irrigação. No entanto, Villa Nova et al. (2003) observaram que para um dado valor da cobertura vegetal, um aumento da evaporação irá proporcionar uma diminuição do Kc, comum em situações de elevada demanda evaporativa da atmosfera em função das restrições impostas pela planta.

Observou-se durante o período experimental que à medida que a cobertura vegetal foi incrementada pelo crescimento da cultura, ocorreu uma diminuição no processo evaporativo do solo, reduzindo o processo de evapotranspiração. Silva e Rao (2006), estudando o consumo hídrico do amendoim em Rodelas, Bahia, encontraram um menor consumo hídrico no inicio da fase de crescimento vegetativo, com aumento nas taxas do final desta mesma fase, devido à alta taxa evapotranspiratória, possivelmente, pelo máximo índice de área foliar atingido pela cultura e pela pronunciada demanda evaporativa da região, em associação aos efeitos de advecção do ar quente e seco. Todavia, Miranda et al. (2007), estudando a demanda hídrica do coqueiro-anão no Ceará, encontraram que houve um aumento contínuo da evapotranspiração do coqueiro em relação à ETo, durante todo o período do estudo, seguindo o aumento da cobertura do solo pela cultura. O Kc médio observado para o quiabeiro durante o estádio de estabelecimento da cultura foi de 0,68.

No Estádio 2 (40 DAP a 70 DAP), caracterizado como período de desenvolvimento vegetativo, observou-se uma variação nos valores de $\mathrm{Kc}$ de 0,5 a 1,03, sendo que o valor médio foi de 0,79 . No final deste período ocorreu o início do florescimento da cultura. Também neste estádio foi observado um incremento progressivo nos valores da área foliar do quiabeiro, como pode ser verificado na Figura 3.

Observa-se que os maiores valores da área foliar (3.350 a $3.470 \mathrm{~cm}^{2}$ planta $^{-1}$ ) foram identificados próximos aos 70 DAP, durante a fase de desenvolvimento vegetativo. Durante este período também foi observado o Kc máximo da cultura (1,03). Carvalho et al. (2007), estudando a demanda hídrica da melancia sem sementes, encontraram, tal qual neste estudo para o quiabeiro, valor máximo do Kc no estádio de desenvolvimento vegetativo, sendo os valores reduzidos no estádio final.

No Estádio 3, caracterizado como estádio de maturação da cultura, o Kc obteve uma variação nos seus valores de 1,0 a 0,3, sendo o valor médio de 0,54. Neste estádio foi possível observar a distinção de duas fases. A primeira fase foi denominada de fase 3A (70 aos $120 \mathrm{DAP}$ ), onde ocorreu um drástico decréscimo da área foliar, ocasionada pelo estabelecimento da fase reprodutiva. $\mathrm{O}$ valor do $\mathrm{Kc}$ da fase $3 \mathrm{~A}$ decresceu de 1,0 para 0,5. A outra fase foi denominada de fase 3B, (120 aos 180 DAP), a qual o Kc obteve variação em seus valores de 0,5 a 0,3 , sendo observado uma tendência na estabilização dos mesmos, assim como dos valores da

Figura 3 - Coeficiente da cultura e a área foliar do quiabeiro

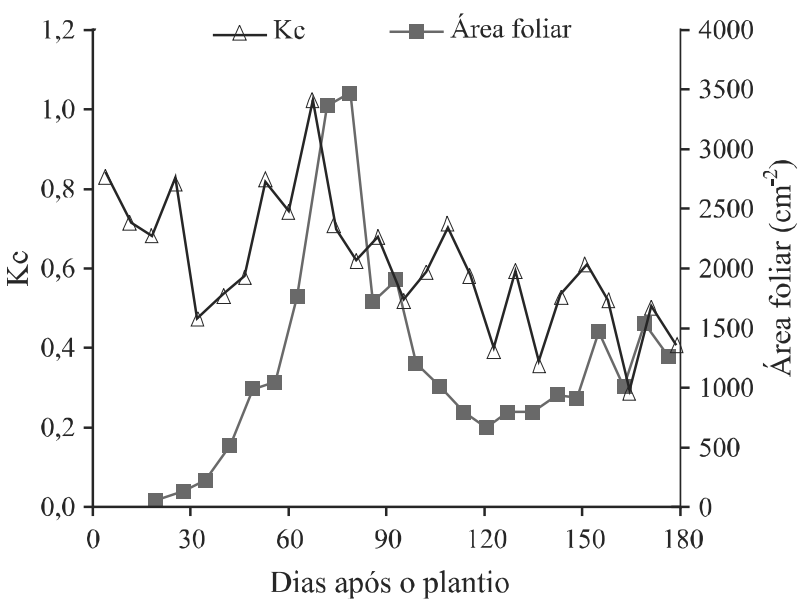


área foliar do quiabeiro. Chaves et al. (2005), estudando a pimenteira, também observaram decréscimo no Kc nas fases de maturação e na colheita. O rendimento obtido na lavoura da área experimental foi de $11,3 \mathrm{Mg} \mathrm{ha}^{-1}$. Segundo Ferreira et al. (2001), a produtividade média na região de Campos dos Goytacazes é de 9,0 $\mathrm{Mg} \mathrm{ha}^{-1}$.

\section{Conclusões}

1. O estádio de estabelecimento da cultura (estádio 1) foi de 40 dias; o estádio de desenvolvimento vegetativo (estádio 2) obteve a duração de 30 dias; o estádio de maturação (estádio 3) foi de 110 dias, sendo dividido em duas fases, $3 \mathrm{~A}$ e $3 \mathrm{~B}$, a primeira com 50 dias e a segunda com 60 dias;

2. O Kc do quiabeiro no primeiro estádio de desenvolvimento variou entre os valores de 0,8 e 0,5 , obtendo o valor médio de 0,68; no segundo estádio variou de 0,5 a 1,03 , sendo a média de 0,79 ; e no terceiro estádio o Kc variou de 1,0 a 0,3, sendo a média de 0,54 ;

3. A evapotranspiração da cultura do quiabeiro no período foi de $314 \mathrm{~mm}$.

\section{Agradecimentos}

Ao CNPq pelo apoio financeiro.

\section{Referências}

ACOSTA, J. F. Consumo Hídrico da Cultura do Girassol Irrigada na Região da Chapada do Apodi - RN. 2009. 73 f. Dissertação (Mestrado em Meteorologia) - Universidade Federal de Campina Grande, Campina Grande.

ALLEN, R. G. et al. Crop evapotranspiration: guidelines for computing crop water requirements. Rome: FAO, 1998. 300 p. (Irrigation and Drainage Paper, 56).

BILIBIO, C. et al. Desenvolvimento vegetativo e produtivo da berinjela submetida a diferentes tensões de água no solo. Revista Brasileira Engenharia Agrícola e Ambiental, v.14, n. 07, p. 730-735, 2010.

CARDOSO, G. B. et al. Determinação da ET de referência pela razão de Bowen com psicrômetros instalados a diferentes alturas. Revista Ciência Agronômica, V. 36, n. 01, p. 16-23, 2005.

CARVAlHO, L. C. C.; BEZERRA, F. M. L.; CARVALHO, M. A. R. Evapotranspiração e coeficientes de cultivo da melancia sem sementes. Revista Ciência Agronômica, v. 39, n. 01, p. 53-59, 2007.
CAVALCANTE, L. F. et al. Teores foliares de macronutrientes em quiabeiro cultivado sob diferentes fontes e níveis de matéria orgânica. Ciências Agrárias, v. 31, n. 01, p. 19-28, 2010.

CHAVES, S. W. P. et al. Evapotranspiração e coeficiente de cultivo da pimenteira em lisímetro de drenagem. Revista Ciência Agronômica, v. 36, n. 03, p. 262-267, 2005

ESTEVES, B. S. et al. Avaliação do Kt para Estimativa da Evapotranspiração de Referência (ETo) em Campos dos Goytacazes, RJ. Brasil. Revista Brasileira de Engenharia Agrícola e Ambiental, v. 14, n. 03, p. 274-278, 2010.

FERREIRA, J. M. et al. Adubação Orgânica e Mineral de Hortaliças no Norte Fluminense. Cultura do Quiabeiro. Horticultura Brasileira, v. 19, Suplemento CD-ROOM, 2001.

HENRIQUE, F. A. N; DANTAS, R. T. Estimativa da evapotranspiração de referencia em Campina Grande, Paraíba. Revista Brasileira de Engenharia Agrícola e Ambiental, v. 11, n. 06, p. 594-599, 2007.

MAROUELLI, W. A.; SILVA, W. L. C. Irrigação por gotejamento do tomateiro industrial durante o estádio de frutificação, na região de Cerrado. Horticultura Brasileira, v. 24, n. 03, p. 342-346, 2006.

MAROUELLI, W. A. et al. Manejo de irrigação em hortaliças 5 ed. Gama: EMBRAPA - CNPH. Revisão ampliada, 1996. 72 p.

MEDEIROS, G. A.; ARRUDA, F. B.; SAKAI, E. Relações entre o coeficiente de cultura e cobertura vegetal do feijoeiro: erros envolvidos e análises para diferentes intervalos de tempo. Acta Scientiarum, v. 26, n. 04, p. 513-519, 2004.

MENDONÇA, J. C. et al. Determinação do coeficiente cultural (Kc) do feijoeiro (Phaseolus vulgaris L.), em Campos dos Goytacazes, RJ. Revista Brasileira de Engenharia Agrícola e Ambiental, v. 11, n. 05, p. 471-475, 2007.

MIRANDA, F. R. et al. Evapotranspiração e coeficientes de cultivo do coqueiro anão-verde na região litorânea do Ceará. Revista Ciência Agronômica, v. 38, n. 02, p. 129-135, 2007.

PAES, H. M. F. Demanda hídrica e função de produção da cultura do quiabeiro (Abelmoschus esculentus (L.) Moench) em Campos do Goytacazes, RJ. 2003, 57 f. Dissertação (Mestrado em Produção Vegetal) - Universidade Estadual do Norte Fluminense, Campos dos Goytacazes.

POSSE, R. P. et al. Evapotranspiração e coeficiente do mamoeiro. Engenharia Agrícola Jaboticabal, v. 28, n. 04, p. 681-690, 2008.

SAAD, A. M.; LIBARDI, P. L. Uso prático do tensiômetro pelo agricultor irrigante. São Paulo: IPT, 1992. 27 p.

SILVA, L. C.; RAO, T. V. R. Avaliação de métodos para estimativa de coeficientes da cultura de amendoim. Revista Brasileira de Engenharia Agrícola e Ambiental, v. 10, n. 01, p. 128-131, 2006.

VILLA NOVA, N. A. et al. Evapotranspiração máxima do feijoeiro, cv. goiano precoce, em função do índice de área foliar e da evaporação do tanque classe A. Ciências Exatas Terra, Ciência Agrícola, v. 9, n. 02, p. 41-45, 2003. 